\title{
Knockdown of LMP1-induced miR-155 sensitizes nasopharyngeal carcinoma cells to radiotherapy in vitro
}

\author{
YUSHENG WANG and LE SUN \\ Department of Otolaryngology, Head and Neck Surgery, The First Hospital of Jilin University, \\ Changchun, Jilin 130021, P.R. China
}

Received December 7, 2014; Accepted December 10, 2015

DOI: $10.3892 / \mathrm{ol} .2016 .4400$

\begin{abstract}
The present study aimed to confirm the promotion of microRNA (miR)-155 expression by latent membrane protein 1 (LMP1), and to recognize the oncogenic role of LMP1 and LMP1-promoted miR-155 in nasopharyngeal carcinoma (NPC), particularly the influence of miR-155 knockdown on the radiosensitivity of CNE-2 cells. Following the regulation of the levels of LMP1 or miR-155 and/or subsequent to radiation treatment, the proliferation ability of CNE-2 cells was examined using 3-(4,5-dimethylthiazol-2-yl)-2,5-diphenyltetrazolium bromide, colony formation and Cell Counting Kit- 8 assays. The results demonstrated that miR-155 was upregulated by overexpression of LMP1 in CNE-2 cells, and LMP1 overexpression and miR-155 mimic transfection increased CNE-2 cell proliferation, whereas miR-155 knockdown attenuated the promotion of CNE-2 cell growth induced by LMP1 overexpression. Furthermore, knockdown of miR-155 enhanced the radiosensitivity of $\mathrm{CNE}-2$ cells. In conclusion, the present study confirmed the oncogenic role of miR-155 in NPC, and demonstrated that knockdown of miR-155 inhibited the growth of NPC cells and sensitized NPC cells to radiotherapy.
\end{abstract}

\section{Introduction}

Nasopharyngeal carcinoma (NPC) is a common malignancy that develops in the head and neck region, and occurs primarily in southern China, Southeast Asia and North Africa, while it is rare in other parts of the world (1). It has been demonstrated that genetic and infectious factors are associated with NPC. Epithelial cells of NPC tumors carry Epstein-Barr virus (EBV) genetic material, and EBV infection has been reported to be associated with $90 \%$ of NPC cases (2). Numerous studies have confirmed that $\mathrm{EBV}$-encoded proteins, including latent

Correspondence to: Dr Yusheng Wang, Department of Otolaryngology, Head and Neck Surgery, The First Hospital of Jilin University, 71 Xinmin Street, Changchun, Jilin 130021, P.R. China E-mail: yshwang2013@163.com

Key words: nasopharyngeal carcinoma, microRNA-155, latent membrane protein 1 , radiotherapy membrane protein 1 (LMP1), BamHI-A rightward frame-1, Epstein-Barr virus nuclear antigen (EBNA)1 and EBNA2, are essential factors in EBV-induced NPC cell transformation, and have been demonstrated to be overexpressed in EBV-associated NPC (3). In particular, the expression of LMP1, considered to be the principal carcinogenic protein of EBV, is positively associated with metastasis of NPC and serves as a good diagnostic marker for NPC $(3,4)$. However, it remains to be elucidated whether these carcinogenic molecules, including LMP1, may be useful as therapeutic targets for the treatment of NPC.

MicroRNAs (miRs) are endogenous short non-coding RNAs of 18-24 nucleotides in length that regulate gene expression in various biological and metabolic processes (5). miRs have a significant role in the regulation of gene expression via degradation of target messenger (m)RNAs or inhibition of translation of target proteins (6). Previous studies focusing on the regulatory effects of miRs in NPC have recognized several miRs that are closely associated with tumorigenesis and progression of NPC. miR-30a (7) and other miRs promote proliferation, invasiveness and metastasis in vitro and/or in vivo via various targets and through various mechanisms, resulting in poor survival of patients with NPC. By contrast, there are miRs that serve as potential tumor suppressors in NPC, including miR-9 (8). Furthermore, certain deregulated miRs in NPC have been reported to be induced by EBV (5). Oncogenic miRs, including miR-10b (9) have been recognized to be induced by or be associated with EBV infection. In addition, EBV infection induces cellular expression of miR-155 in NPC (10), and upregulated miR-155 during EBV infection was promoted by expression of EBV-encoded LMP1 (10).

In the present study, in order to confirm the promotion of miR-155 by LMP1, and to recognize the oncogenic role of LMP1 and LMP1-promoted miR-155 in NPC, an LMP1-overexpressing CNE-2 cell line was constructed, and miR-155 upregulation was examined in this cell line. Subsequently, the regulatory role of LMP1 and miR-155 on cell proliferation was investigated. Furthermore, the influence of knockdown of LMP1-induced miR-155 on the sensitivity of CNE-2 cells to radiation treatment was assessed.

\section{Materials and methods}

Cell culture, LMP1 overexpression and miR-155 manipulation. NPC CNE-2 cells were purchased from the Cell Resource 
Center of the Chinese Academy of Medical Sciences (Beijing, China). Cells were grown or maintained in RPMI-1640 medium (catalog no., 31800-022; Thermo Fisher Scientific, Inc., Waltham, MA, USA), which was supplemented with $10 \%$ (for growth) or $2 \%$ (for maintenance) fetal bovine serum (catalog no., 1009-141-FBS; Gibco ${ }^{\circledR}$; Thermo Fisher Scientific, Inc.), $50 \mu \mathrm{g} / \mathrm{ml}$ penicillin (catalog no., P7794; Sigma-Aldrich, St. Louis, MO, USA) and $50 \mu \mathrm{g} / \mathrm{ml}$ streptomycin (catalog no., P4333; Sigma-Aldrich). Cells were incubated at $37^{\circ} \mathrm{C}$ in an atmosphere of $5 \% \mathrm{CO}_{2}$. For transient LMP1 overexpression, LMP1-pcDNA3.1 recombinant plasmid (10) was transfected into CNE-2 cells using Lipofectamine ${ }^{\circledR} 2000$ (catalog no., 12566014; Invitrogen ${ }^{\circledR}$; Thermo Fisher Scientific, Inc.), at a concentration of $0,0.2,0.5$ or $1 \mu \mathrm{g} / \mathrm{ml}$ for 12 (for LMP1 messenger (m)RNA assay), 24 (for LMP1 protein, cell viability and cell proliferation assays), 48 or $72 \mathrm{~h}$ (for cell proliferation assay). For sustained LMP1 overexpression, CNE-2 cells were transfected with the aforementioned LMP1-pcDNA3.1 recombinant plasmid, and cultured under Geneticin ${ }^{\circledR}$ (G418; catalog no., 11811023; Thermo Fisher Scientific, Inc.) pressure $(800 \mu \mathrm{g} / \mathrm{ml})$. The positive cell clones were propagated in RPMI-1640 medium containing $500 \mu \mathrm{g} / \mathrm{ml} \mathrm{G} 418$. To manipulate the levels of miR-155, CNE-2 cells were transfected with miR-155 mimic or miR-155 inhibitor (Qiagen, Inc., Valencia, CA, USA) using Lipofectamine 2000, while miR-Con was utilized as a control miRNA.

RNA extraction and reverse transcription-quantitative polymerase chain reaction $(R T-q P C R)$. Total cellular mRNA was isolated and extracted from CNE-2 cells using RNeasy Mini kit (catalog no., 74104; Qiagen GmbH, Hilden, Germany.). The sample was purified using the RNase-Free DNase Set (catalog no., 79254; Qiagen, Inc.) according to the manufacturer's instructions. RT-qPCR analysis of the mRNA levels of LMP1 in CNE-2 cells was performed with One-Step SYBR PrimeScript RT-PCR Kit II (Perfect Real Time) (catalog no., RR086A/B; Takara Bio, Inc., Otsu, Japan) using a quantitative PCR instrument (LightCycler ${ }^{\circledR}$ 2.0; Roche Applied Science, Penzberg, Germany) and the following primers, which were designed by Primer Express 2 software (Applied Biosystems; Thermo Fisher Scientific, Inc.) and synthesized by Sangon Biotech Co., Ltd., (Shanghai, China): Forward, 5'-GCAGCCTCACGCACATCGA-3' and reverse, 5'-GGGAGGCGCTTGGTGCAAA-3' for LMP-1; and forward, 5'-TGCACCACCAACTGCTTAG-3' and reverse, 5'-TCTGGGTGGCAGTGAT-3' for glyceraldehyde-3-phosphate dehydrogenase (GAPDH). RT-qPCR was performed under the following conditions: Denaturation at $42^{\circ} \mathrm{C}$ for $8 \mathrm{~min}, 95^{\circ} \mathrm{C}$ for $30 \mathrm{sec}$, followed by 40 cycles of $95^{\circ} \mathrm{C}$ for $10 \mathrm{sec}$ and at $60^{\circ} \mathrm{C}$ for $30 \mathrm{sec}$. The RNA expression levels were normalized to the levels of GAPDH. miR in CNE-2 cells was extracted using mirVana ${ }^{\mathrm{TM}}$ miRNA Isolation kit (catalog no., Am1561; Ambion ${ }^{\circledR}$; Thermo Fisher Scientific, Inc.). Quantitative analysis of the levels of miR-155 in CNE-2 cells was conducted with mirVana ${ }^{\mathrm{TM}}$ qRT-PCR miRNA Detection kit (catalog no., Am1558; Ambion; Thermo Fisher Scientific), using the following primers: 5'-UUAAUGCUAAUUGUG AUAGGGGU-3' for miR-155; 5'-GCAGGGGAACUCAUC AUCUCUG-3' for U6 spliceosomal RNA; and 5'-UUUCAUCCUUGUGCAGGG-3' for universal primer. qPCR was performed under the following conditions: Denaturation at $95^{\circ} \mathrm{C}$ for $15 \mathrm{~min}$, followed by 40 cycles of $94^{\circ} \mathrm{C}$ for $15 \mathrm{sec}$, $55^{\circ} \mathrm{C}$ for $15 \mathrm{sec}$ and $70^{\circ} \mathrm{C}$ for $20 \mathrm{sec}$. U6 spliceosomal RNA served as an internal control. The $\Delta \Delta \mathrm{Cq}$ method was utilized for relative quantification (11).

Protein sample isolation and western blot analysis. Protein samples were extracted from CNE-2 cells with M-PER ${ }^{\mathrm{TM}}$ Mammalian Protein Extraction Reagent (catalog no., 78501; Thermo Fisher Scientific, Inc.). Subsequently, each protein sample was separated using 8 or $12 \%$ sodium dodecyl sulfate-polyacrylamide gel electrophoresis, prior to being transferred onto a nitrocellulose membrane. The membranes were blocked at $4^{\circ} \mathrm{C}$ overnight with Tris-buffered saline and Tween 20 (50 mM Tris-HCl, pH 7.5; 150 mM NaCl; 0.05\% Tween 20; catalog no., 9005-64-5; Sigma-Aldrich) containing $5 \%$ skimmed milk, prior to be incubated with mouse monoclonal EBV LMP1 (catalog no., sc-57721; 1:300; Santa Cruz Biotechnology Inc., Santa Cruz, CA, USA) or rabbit anti-human polyclonal GAPDH (catalog no., 100242-T40-50; 1:1,000; Sino Biological, Inc., Beijing, China) primary antibodies for $2 \mathrm{~h}$ at room temperature, followed by incubation with goat anti-rabbit oligoclonal immunoglobulin $\mathrm{G}$ conjugated to horseradish peroxidase (catalog no., A27036; 1:1,000; Thermo Fisher Scientific, Inc.) at $37^{\circ} \mathrm{C}$ for $45 \mathrm{~min}$. The target protein band was visualized using the enhanced chemiluminescence detection system (SuperSignal West Femto Maximum Sensitivity Substrate; catalog no., 32209; Pierce $^{\circledR}$; Thermo Fisher Scientific, Inc.). Protein bands were quantified using ImageJ 1.45 software (http://imagej.nih. gov/ij/).

Determination of cell viability and cell proliferation by 3-(4,5-dimethylthiazol-2-yl)-2,5-diphenyltetrazolium bromide (MTT), Cell Counting Kit (CCK)-8 and cell colony formation assays. Cell viability was determined via MTT assay (catalog no., KA1606; Invitrogen; Thermo Fisher Scientific, Inc.). Cells seeded into 96-well plates (Corning Inc., Tewksbury, MA, USA) were transfected with $0.00,0.25,0.50,0.75$ or $1.00 \mu \mathrm{g} / \mathrm{ml}$ LMP1-pcDNA3.1 or control pcDNA3.1 for $24 \mathrm{~h}$. In another experiment, the seeded CNE-2 (LMP1 or Con) cells were transfected with 0,20 or $40 \mathrm{nM}$ miR-155 inhibitor or miR-Con, respectively, and were irradiated with $0,2,4,6$ or 8 Gy (RS225 X-Ray Research System; Gulmay Medical Ltd., Surrey, UK). Subsequently, MTT assay was conducted according to the standard protocol (12). Absorbance was measured at $570 \mathrm{~nm}$ with a reference wavelength of $750 \mathrm{~nm}$ using a spectrophotometer (F-2000; Hitachi, Tokyo, Japan). For the cell proliferation assay, CNE-2 (LMP1), CNE-2 (Con) or CNE-2 cells subjected or not to transfection with miR-155 inhibitor or miR-155 control, were incubated in the presence of CCK-8 (catalog no., CK04; Dojindo Molecular Technologies, Inc., Kumamoto, Japan). The absorbance at $450 \mathrm{~nm}$ of each well was detected following visual color occurrence at $24 \mathrm{~h}$. All experiments were performed in triplicate. For the cell colony formation assay, $5 \times 10^{2}$ cells were incubated in 6-well plates (Corning Inc.) at $37^{\circ} \mathrm{C}$ in an atmosphere of 5\% $\mathrm{CO}_{2}$. At 3-6 days post-incubation, the cells were stained using crystal violet $(0.005 \%$; catalog no., C3886; Sigma-Aldrich) for $20 \mathrm{~min}$, and colony numbers were recorded using ImageJ 1.45 software (http://imagej.nih.gov/ij/). 
A

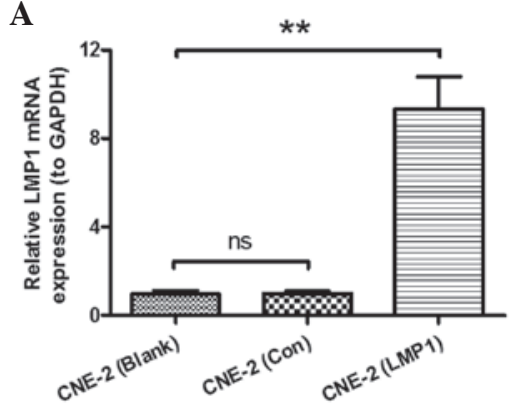

D

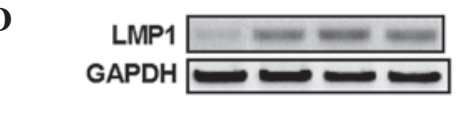

ns

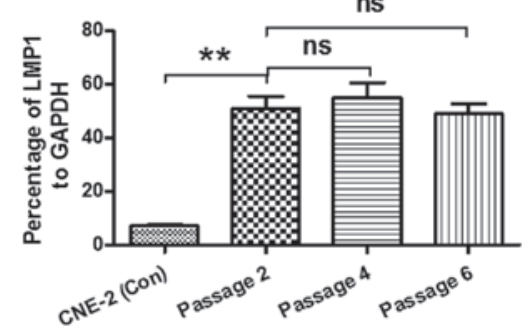

B

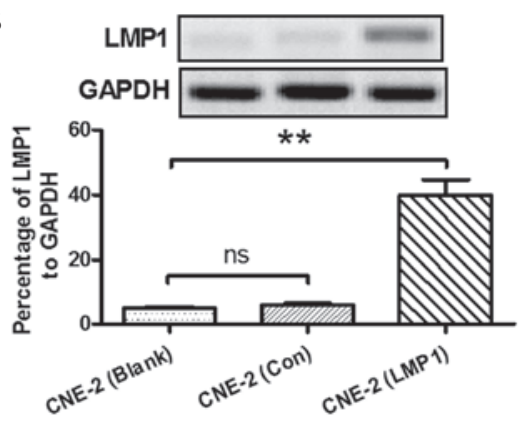

E

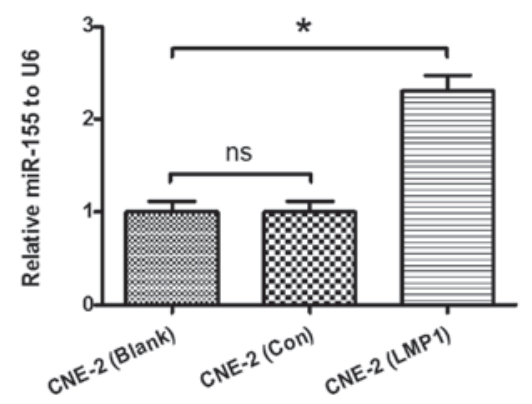

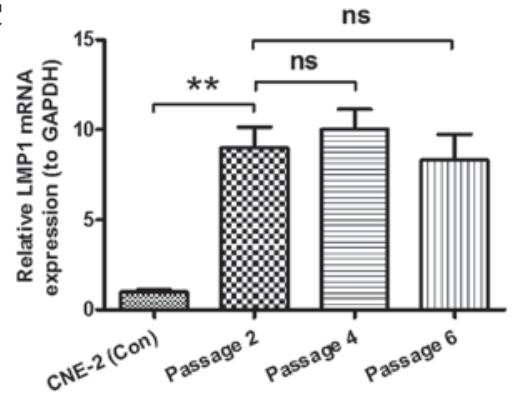

F

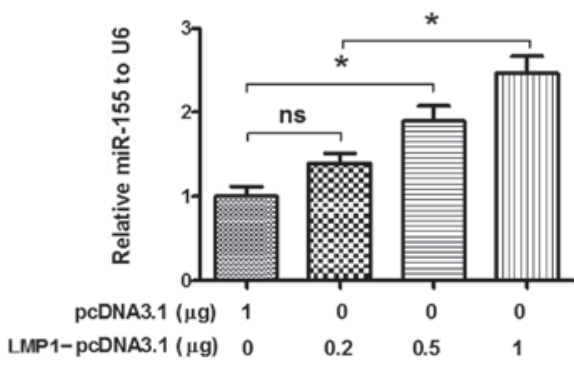

Figure 1. LMP1 of Epstein-Barr virus promotes miR-155 expression in CNE-2 cells. CNE-2 cells were separated into three groups: Non-transfected CNE-2 cells (Blank), CNE-2 cells transfected with empty pcDNA3.1 as negative control (Con) and LMP1-pcDNA3.1-transfected CNE-2 cells (LMP1). (A) Relative mRNA expression levels of LMP1 in the three groups of CNE-2 cells, compared with the levels of GAPDH. (B) Percentage of LMP1 expression at the protein level in the three CNE-2 cell groups, as revealed by western blot analysis. (C) Relative mRNA levels of LMP1 vs. GAPDH in the three groups of CNE-2 cells upon a number of serial passages, as determined by reverse transcription-quantitative polymerase chain reaction. (D) Overexpression of LMP1 protein in CNE-2 cells following a number of serial passages. (E) Relative miR-155 levels in the three groups of CNE-2 cells, compared with the levels of U6 snRNA. (F) Relative miR-155 levels in CNE-2 cells transfected with various concentrations of LMP1-pcDNA3.1, compared with the levels of U6 snRNA. * $<0.05$, ${ }^{* *} \mathrm{P}<0.01$. LMP1, latent membrane protein 1; miR, microRNA; mRNA, messenger RNA; GAPDH, glyceraldehyde-3-phosphate dehydrogenase; ns, not significant; Con, control; snRNA, spliceosomal RNA.

Statistical evaluations. Results are expressed as the mean \pm standard error. Student's t-tests were performed to compare the differences between two groups. Statistical analyses were performed using GraphPad Prism 6.0 software (GraphPad Software, Inc., La Jolla, CA, USA). P<0.05 was considered to indicate a statistically significant difference.

\section{Results}

miR-155 expression is upregulated by overexpression of LMPI in CNE-2 cells. A previous study by the present group revealed that expression of miR-155 was upregulated in CNE-2 cells following transfection with an LMP1-expressing plasmid (10). In order to further confirm the association between miR-155 expression and overexpression of LMP1, RT-qPCR was performed in the present study to detect miR-155 expression. LMP1 expression in the CNE-2 (LMP1) group exhibited a significant difference in terms of mRNA $(\mathrm{P}<0.01$; Fig. 1A) and protein levels $(\mathrm{P}<0.01$; Fig. 1B), compared with the other groups. Furthermore, as shown in Fig. 1C and D, the expression levels of LMP1 in the CNE-2 (LMP1) group demonstrated no significant difference between the two serial passages, and was significantly increased, compared with the CNE-2 (Con) group $(\mathrm{P}<0.01)$. Overexpression of LMP1 in the CNE-2 cell line revealed a significant increase in the expression of miR-155, as demonstrated in Fig. 1E $(\mathrm{P}<0.05)$. Furthermore, the transfection efficiency of LMP1-pcDNA3.1 plasmid was detected. As shown in Fig. 1F, $>0.5 \mu \mathrm{g}$ of LMP1-pcDNA3.1 was a concentration that effectively increased the expression levels of miR-155 in CNE-2 cells $(\mathrm{P}<0.05)$. These results suggested that overexpression of LMP1 was able to upregulate the expression of miR-155 in CNE-2 cells.

LMP1 overexpression and miR-155 mimic transfection increase the proliferation of CNE-2 cells. MTT assay was performed in order to assess the effect of LMP1 overexpression on the viability of the CNE-2 cell line. For this purpose, cells were transfected with LMP1-pcDNA3.1 or pcDNA3.1 control, according to the manufacturer's protocol, using a series of concentrations ranging from 0.00 to $1.00 \mu \mathrm{g}$. The viability of LMP1-pcDNA3.1-transfected cells was significantly increased compared with pcDNA3.1-transfected cells, as shown in Fig. 2A $(\mathrm{P}<0.05)$. Subsequently, cell proliferation following pcDNA3.1 or LMP1-pcDNA3.1 transfection was detected by CCK-8 assay in the two groups of CNE-2 cells. As demonstrated in Fig. 2B, the CNE-2 (LMP1) cells exhibited significantly increased proliferation compared with the CNE-2 (Con) cells $(\mathrm{P}<0.05 ; \mathrm{P}<0.01)$. Furthermore, the proliferation of CNE-2 cells transfected with miR-155 mimic or miR-Con was additionally examined. The expression levels of miR-155 in CNE-2 cells transfected with miR-155 mimic were significantly increased $(\mathrm{P}<0.001$; Fig. $2 \mathrm{C})$. In addition, CCK-8 assay demonstrated that transfection with 20 or $40 \mathrm{nM}$ miR-155 mimic significantly promoted CNE-2 cell proliferation $(\mathrm{P}<0.05$; Fig. 2D). The results of the present 
A

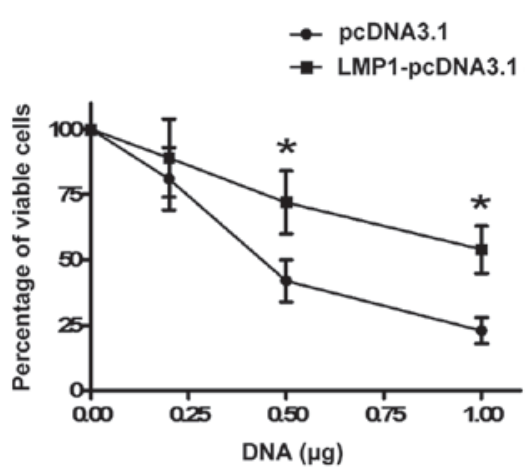

C

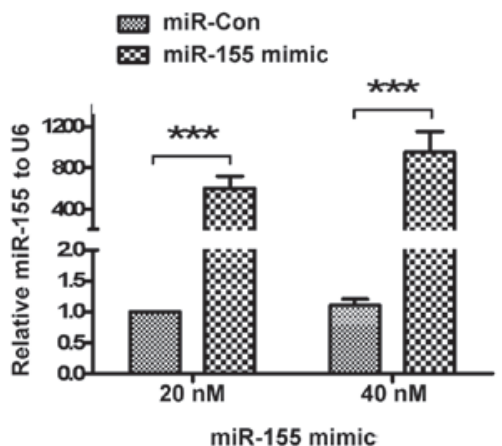

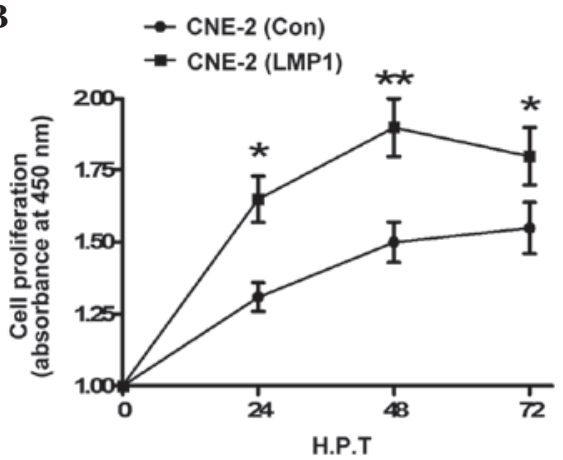

D

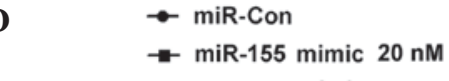

+ miR-155 mimic $40 \mathrm{nM}$

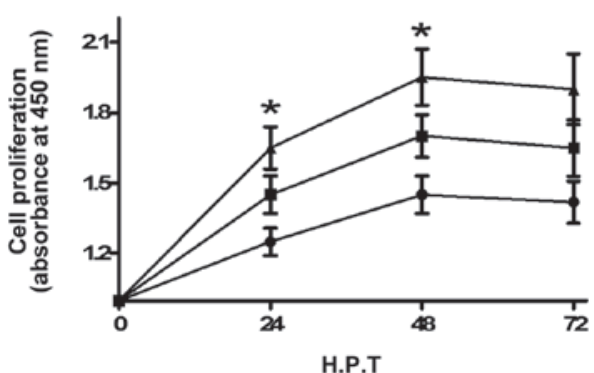

Figure 2. LMP1 promotes the viability of CNE-2 cells by inducing miR-155. (A) CNE-2 cell viability following transfection with various concentrations of empty pcDNA3.1 or LMP1-pcDNA3.1 vectors, as revealed by 3-(4,5-dimethylthiazol-2-yl)-2,5-diphenyltetrazolium bromide assay. (B) Growth curve of cell proliferation following transfection with empty pcDNA3.1 or LMP1-pcDNA3.1 vectors. (C) miR-155 levels in CNE-2 cells transfected with miR-Con or miR-155 mimic. (D) Growth curve of cell proliferation following transfection with miR-Con or miR-155 mimic (20 or $40 \mathrm{nM})$ by Cell Counting Kit- 8 assay. ${ }^{*} \mathrm{P}<0.05,{ }^{* *} \mathrm{P}<0.01,{ }^{* * *} \mathrm{P}<0.001$. LMP1, latent membrane protein 1; miR, microRNA; Con, control; H.P.T., hours post-transfection.

A

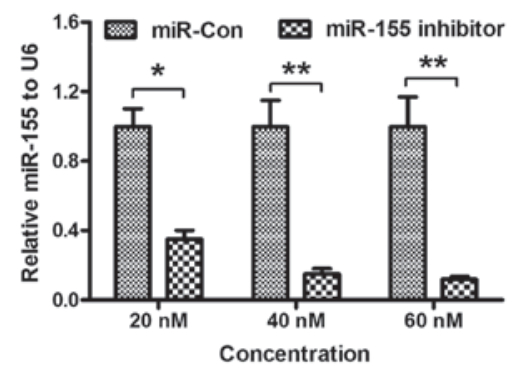

B

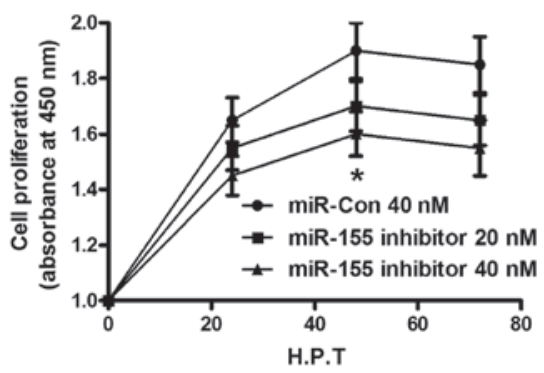

C

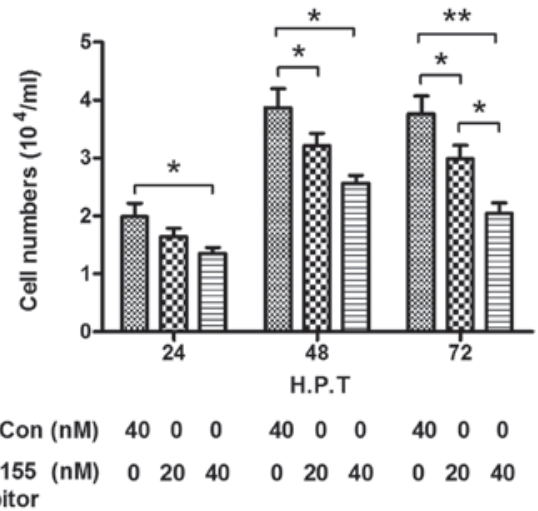

Figure 3. miR-155 inhibitor suppresses LMP1-promoted CNE-2 cell proliferation. (A) miR-155 expression was inhibited significantly in CNE-2 cells transfected with miR-155 inhibitor. Cell proliferation was suppressed following transfection with miR-155 inhibitor, as revealed by (B) 3-(4,5-dimethylthiazol-2-yl)-2,5-diphenyltetrazolium bromide and (C) Cell Counting Kit-8 assays. "P<0.05; ${ }^{* *} \mathrm{P}<0.01$. miR, microRNA; LMP1, latent membrane protein 1; Con, control; H.P.T., hours post-transfection.

study confirmed that LMP1 overexpression or miR-155 mimic transfection promoted the proliferation of CNE-2 cells.

miR-155 knockdown attenuates the promotion of cell growth in CNE-2 cells caused by overexpression of LMP1. To further investigate the promotion of CNE- 2 cell growth by miR-155, the expression of miR-155 in CNE-2 cells was knocked down by addition of miR-155 inhibitor, and the influence of miR-155 knockdown on the growth of CNE-2 cells was determined. The results indicated that transfection with 20 , 40 or $60 \mathrm{nM}$ miR-155 inhibitor significantly reduced the levels of miR-155 in CNE-2 cells, compared with miR-Con cells $(\mathrm{P}<0.05 ; \mathrm{P}<0.01$; Fig. $3 \mathrm{~A})$. Furthermore, cell proliferation demonstrated a significant decrease upon transfection with miR-155 inhibitor (20 or $40 \mathrm{nM}$ ), as shown in Fig. 3B $(\mathrm{P}<0.05)$. In addition, the results of CCK- 8 assay indicated that the cell number in the miR-155 inhibitor group was significantly reduced, compared with the miR-Con group $(\mathrm{P}<0.05 ; \mathrm{P}<0.01 ;$ Fig. $3 \mathrm{C})$. The results of the present study 
A
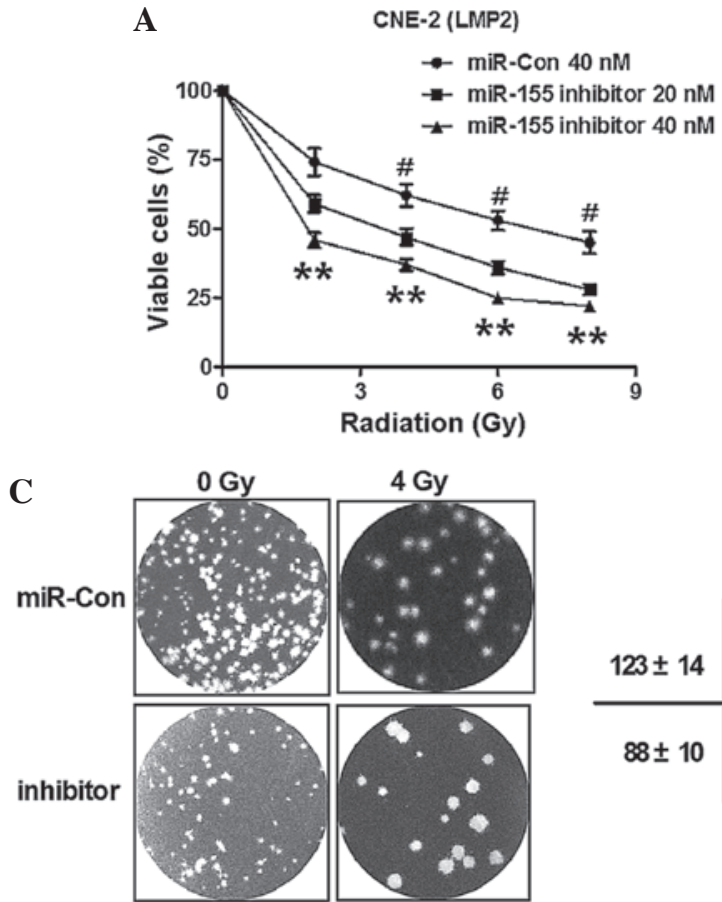
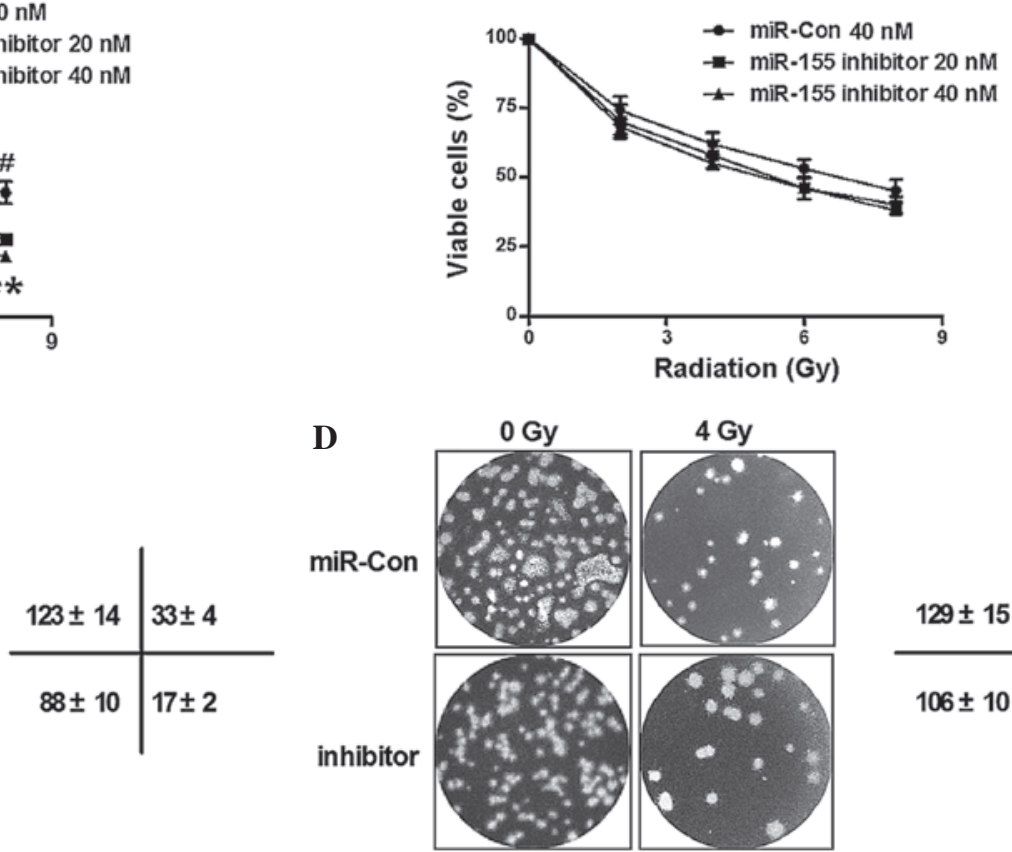

B

D

Radiation (Gy)

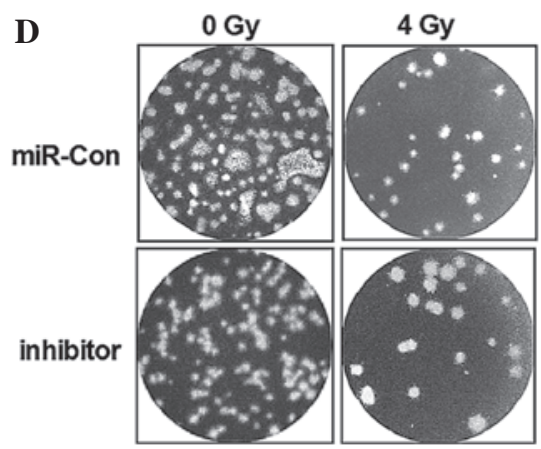

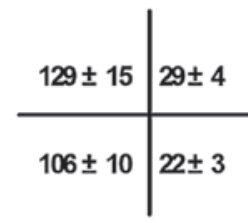

Figure 4. Blockage of LMP1-induced miR-155 sensitizes CNE-2 cells to radiotherapy in vitro. (A) miR-155 inhibitor enhanced the radiation-induced viability reduction of CNE-2 (LMP1) cells. (B) miR-155 inhibitor had no influence on the sensitivity of normal CNE-2 cells to radiation. (C and D) Difference in cell growth subsequent to radiation treatment in CNE-2 cells transfected with miR-Con vs. CNE-2 cells transfected with miR-155 inhibitor, as detected by colony formation assay for (C) CNE-2 (LMP1) cells and (D) normal CNE-2 cells. ${ }^{*} \mathrm{P}<0.05 ;{ }^{* *} \mathrm{P}<0.01$. LMP1, latent membrane protein 1; Con, control; miR, microRNA.

confirmed that miR-155 knockdown inhibited the promotion of proliferation of CNE-2 cells caused by miR- 155 .

Knockdown of LMPI-induced miR-155 enhances the radiosensitivity of CNE-2 cells. Radiotherapy is widely perceived to be the most effective treatment for NPC $(13,14)$. In order to investigate whether miR-155 served as an alternative target to sensitize NPC cells to radiotherapy, the radiosensitivity of CNE-2 cells post-miR-155 knockdown was determined. Initially, the viability of normal CNE-2 cells or CNE-2 (LMP1) cells with miR-155 knockdown post-radiation was determined. As shown in Fig. 4A, cell viability was reduced when miR-155 was blocked in CNE-2 cells overexpressing LMP1 $(\mathrm{P}<0.05$; $\mathrm{P}<0.01)$. By contrast, the viability was not significantly influenced in the CNE-2 (Con) cells post-transfection with miR-155 inhibitor ( $\mathrm{P}>0.05$; Fig. 4B). Furthermore, colony formation assay was performed for CNE-2 (LMP1) cells and CNE-2 (Con) cells, with or without miR-155 knockdown, following treatment with 0 or $4 \mathrm{~Gy}$ radiation. As demonstrated in Fig. $4 \mathrm{C}$, there were less colonies formed by CNE-2 (LMP1) cells upon transfection with miR-155 inhibitor, compared with miR-Con transfected cells $(123 \pm 14$ vs. $88 \pm 10$ cells, respectively; $\mathrm{P}<0.01)$. Furthermore, the colony number reduction by miR-155 knockdown was more significant in CNE-2 (LMP1) cells transfected with miR-155 inhibitor than in miR-Con-transfected cells ( $33 \pm 4$ vs. $17 \pm 2$ cells, respectively; $\mathrm{P}<0.01$ ). However, the colony number reduction caused by miR-155 knockdown was not significant in CNE-2 (Con) cells, with or without exposure to 4 Gy radiation ( $>0.05$; Fig. 4D). Thus, the results of the present study confirmed that knockdown of LMP1-induced miR-155 decreased the sensitivity of CNE-2 cells to radiation.

\section{Discussion}

EBV infection of primary B cells results in latent infection, in which a subset of viral genes are expressed (15). There are three types of EBV latent infection (16); in particular, NPC correlates with type II latency, in which LMP1 and LMP2 are overexpressed and contribute to oncogenesis (16). The integral transmembrane protein LMP1 potentiates a variety of signaling pathways, including nuclear factor- $\kappa \mathrm{B}$, mitogen-activated protein kinase, phosphoinositide 3-kinase/Akt (17) and eukaryotic translation initiation factor $4 \mathrm{E}$, to promote the proliferation, migration and invasion of NPC (18). Previous studies have revealed deregulated miR expression post-EBV infection in NPC or lymphocytes $(9,19,20)$. Furthermore, a previous study has recognized the deregulation of miRs by LMP1, including miR-10b (9). In a previous study by the present research group, miR-155 was identified to be upregulated by LMP1 DNA, and contributed to the proliferation and migration of NPC cells (10).

In the present study, the CNE-2 NPC cell line was selected to investigate the role of miR-155 induced by LMP1 in the sensitization of NPC cells to radiotherapy. Initially, the promotion of miR-155 expression by LMP1 overexpression was confirmed in CNE-2 cells. Subsequently, it was identified that overexpression of LMP1 and transfection with miR-155 mimic promoted the proliferation of CNE-2 cells, according to the results of MTT and CCK- 8 assays. Furthermore, miR-155 knockdown with miR-155 inhibitor attenuated the promotion of CNE-2 cell growth caused by LMP1, thus confirming the key regulatory role of miR-155 in LMP1-promoted NPC cell 
proliferation. Radiotherapy is currently the most effective treatment for NPC $(13,14)$. The present study confirmed that miR-155 may serve as a novel target for the sensitization of NPC cells to radiotherapy. Based on the results of the cell viability, cell proliferation and colony formation assays, miR-155 knockdown was confirmed to significantly enhance the radiosensitivity of CNE-2 cells.

miR-155 is encoded by the MIR155 host gene, and regulates various physiological and pathological processes (21), including inflammatory processes and various signaling pathways in cancer (21). miR-155 has been considered to act as an oncogene or tumor suppressor, depending on the type of tumor (22). miR-155 has been observed to be oncogenic in NPC (10), but tumor-suppressive in gastric cancer (23). The present study confirmed the oncogenic role of miR-155 in NPC. Furthermore, the strategy to knockdown miR-155 has been previously investigated for the control of cancer progression (24). Previous studies have demonstrated that knockdown of miR-155 in mice resulted in rapid regression of lymphadenopathy, in part due to apoptosis of the malignant lymphocytes (25). In addition, systemic delivery of antisense peptide nucleic acids against miR-155 that were encapsulated in unique polymer nanoparticles inhibited miR-155 and retarded the growth of pre-B-cell tumors in vivo, suggesting a potential therapeutic option for the treatment of lymphoma/leukemia (25). In the present study, it was identified that knockdown of miR-155 inhibited NPC cell growth and sensitized NPC cells to radiotherapy in vitro. These results suggest that downregulation of miR-155 may provide a novel approach for the treatment of NPC.

In conclusion, the present study confirmed the oncogenic role of miR-155 in NPC, and demonstrated that knockdown of miR-155 inhibited the growth of NPC cells and additionally sensitized these cells to radiotherapy.

\section{Acknowledgements}

The present study was supported by a grant from the Development and Reform Commission of Jilin Province (Changchun, China; grant no. 3J113Z363428).

\section{References}

1. Guo X, O'Brien SJ,Zeng Y, Nelson GW and Winkler CA: GSTM1 and GSTT1 gene deletions and the risk for nasopharyngeal carcinoma in Han Chinese. Cancer Epidemiol Biomarkers Prev 17: 1760-1763, 2008

2. Wolf $H$, zur Hausen $H$ and Becker V: EB viral genomes in epithelial nasopharyngeal carcinoma cells. Nat New Biol 244 245-247, 1973.

3. Xu Y, Shi Y, Yuan Q, Liu X, Yan B, Chen L, Tao Y and Cao Y: Epstein-Barr Virus encoded LMP1 regulates cyclin D1 promoter activity by nuclear EGFR and STAT3 in CNE1 cells. J Exp Clin Cancer Res 32: 90, 2013.

4. Zhao Y, Wang Y, Zeng S and Hu X: LMP1 expression is positively associated with metastasis of nasopharyngeal carcinoma: Evidence from a meta-analysis. J Clin Pathol 65: 41-45, 2012.

5. Bartel DP: MicroRNAs: Genomics, biogenesis, mechanism, and function. Cell 116: 281-297, 2004.

6. Niu Q, Qian M, Liu G, Yang F and Teng Y: A genome-wide identification and characterization of microRNAs and their targets in 'Suli' pear (Pyrus pyrifolia white pear group). Planta (Sep) 8, 2013 (Epub ahead of print).
7. Wang HY, Li YY, Fu S, Wang XP, Huang MY, Zhang X, Shao Q, Deng L, Zeng MS, Zeng YX and Shao JY: MicroRNA-30a promotes invasiveness and metastasis in vitro and in vivo through epithelial-mesenchymal transition and results in poor survival of nasopharyngeal carcinoma patients. Exp Biol Med (Maywood) 239: 891-898, 2014.

8. Lu J, Luo H, Liu X, Peng Y, Zhang B, Wang L, Xu X, Peng X, Li G, Tian W, et al: miR-9 targets CXCR4 and functions as a potential tumor suppressor in nasopharyngeal carcinoma. Carcinogenesis 35: 554-563, 2014.

9. Li G, Wu Z, Peng Y, Liu X, Lu J, Wang L, Pan Q, He ML and Li XP: MicroRNA-10b induced by Epstein-Barr virus-encoded latent membrane protein-1 promotes the metastasis of human nasopharyngeal carcinoma cells. Cancer Lett 299: 29-36, 2010.

10. Zhu X, Wang Y, Sun Y, Zheng J and Zhu D: MiR-155 up-regulation by LMP1 DNA contributes to increased nasopharyngeal carcinoma cell proliferation and migration. Eur Arch Otorhinolaryngol 271: 1939-1945, 2014.

11. Livak KJ and Schmittgen TD: Analysis of relative gene expression data using real time quantitative PCR and the 2( Delta Delta C(T)) Method. Methods 25: 402 408, 2001.

12. Freimoser FM, Jakob CA, Aebi M and Tuor U: The MTT [3-(4,5-dimethylthiazol-2-yl)-2,5-diphenyltetrazolium bromide] assay is a fast and reliable method for colorimetric determination of fungal cell densities. Appl Environ Microbiol 65: 3727-3729, 1999.

13. Tang JM, Ma XM, Hou YL, Dai LY, Cao HB, Ye M and Bai YR: Analysis of simultaneous modulated accelerated radiotherapy (SMART) for nasopharyngeal carcinomas. J Radiat Res 55: 794-802, 2014

14. Hua YJ, Han F, Lu LX, Mai HQ, Guo X, Hong MH, Lu TX and Zhao C: Long-term treatment outcome of recurrent nasopharyngeal carcinoma treated with salvage intensity modulated radiotherapy. Eur J Cancer 48: 3422-3428, 2012.

15. Kaneda A, Matsusaka K, Aburatani H and Fukayama M: Epstein-Barr virus infection as an epigenetic driver of tumorigenesis. Cancer Res 72: 3445-3450, 2012.

16. Murata T, Sato Y and Kimura H: Modes of infection and oncogenesis by the Epstein-Barr virus. Rev Med Virol 24: 242-253, 2014.

17. Dawson CW, Tramountanis G, Eliopoulos AG and Young LS: Epstein-Barr virus latent membrane protein 1 (LMP1) activates the phosphatidylinositol 3-kinase/Akt pathway to promote cell survival and induce actin filament remodeling. J Biol Chem 278: 3694-3704, 2003.

18. Zhao Y, Pang TY, Wang Y, Wang S, Kang HX, Ding WB, Yong WW, Bie YH, Cheng XG, Zeng C, et al: LMP1 stimulates the transcription of eIF4E to promote the proliferation, migration and invasion of human nasopharyngeal carcinoma. FEBS J 281: 3004-3018, 2014.

19. Luo Z, Dai Y, Zhang L, Jiang C, Li Z, Yang J, McCarthy JB, She $\mathrm{X}$, Zhang $\mathrm{W}$, Ma J, et al: miR-18a promotes malignant progression by impairing microRNA biogenesis in nasopharyngeal carcinoma. Carcinogenesis 34: 415-425, 2013.

20. Yu H, Lu J, Zuo L, Yan Q, Yu Z, Li X, Huang J, Zhao L, Tang H, Luo Z, et al: Epstein-Barr virus downregulates microRNA 203 through the oncoprotein latent membrane protein 1: A contribution to increased tumor incidence in epithelial cells. J Virol 86: 3088-3099, 2012

21. O'Connell RM, Rao DS and Baltimore D: MicroRNA regulation of inflammatory responses. Annu Rev Immunol 30: 295-312, 2012.

22. Chen Z, Ma T, Huang C, Hu T and Li J: The pivotal role of microRNA-155 in the control of cancer. J Cell Physiol 229: 545-550, 2014.

23. Sun S, Sun P, Wang C and Sun T: Downregulation of microRNA-155 accelerates cell growth and invasion by targeting c-myc in human gastric carcinoma cells. Oncol Rep 32: 951-956, 2014.

24. Mattiske S, Suetani RJ, Neilsen PM and Callen DF: The oncogenic role of miR-155 in breast cancer. Cancer Epidemiol Biomarkers Prev 21: 1236-1243, 2012.

25. Babar IA, Cheng CJ, Booth CJ, Liang X, Weidhaas JB, Saltzman WM and Slack FJ: Nanoparticle-based therapy in an in vivo microRNA-155 (miR-155)-dependent mouse model of lymphoma. Proc Natl Acad Sci USA 109: E1695-E1704, 2012. 